\title{
Electrical Behavior of Connectors under Different Atmospheres
}

\author{
R. El Abdi, J. Labbé, and F. Le Strat
}

\begin{abstract}
Connector was composed of a female and male part which induced vibrations from the vehicle might lead to relative movements between these two parts. A relative movement of the contact zone between these two parts can lead to an irreversible mechanical degradation and an electrical perturbation by the formation of a third-body layer at the contact zone. This was the fretting corrosion phenomenon in electrical contact, which depends of the contact materials used, the atmosphere in which the connectors were used, and mechanical parameters (contact force, material hardness ...). For economic reasons, the tin was used for coating to protect the substrate made of copper. In the air, the oxygen reacts with soft tin and forms a hard and brittle layer of tin oxides at the surface, which become remains under vibration stresses.

Our study is about the measurement of electrical behavior of metallic connectors in two atmospheres: air and nitrogen as an inert gas. When a connector is tested in air atmosphere, a typical increase of the contact voltage amplitude was obtained. On the other hand, a nitrogen gas atmosphere leads to the contact voltage decrease. The change of the contact voltage highlights the ejection and the production of remains which were not so well understand. The study of the contact voltage provides new information about the degradation, to understand the fretting-corrosion phenomena.
\end{abstract}

Index Terms-Automotive connectors, fretting corrosion, electrical contact, nitrogen gas.

\section{INTRODUCTION}

In the automotive fields, the vehicle vibrations induce movement on hundreds of connectors which were located near the engine, inside the seat and at many other zones. The vibrations could induce a displacement between the male and female part i.e. the pin and the clip and could generate an electrical failure due to the well-known fretting-corrosion phenomenon [1]-[8]. A relative displacement of $5 \mu \mathrm{m}$ was enough to produce remains at the interface between the pin and the clip and set an intermittent failure at the interface [9]. This phenomenon represents $60 \%$ of electrical failure within a car [10]. Electrical contacts were made of a substrate of copper alloy plated with a thin protective layer of non-noble metals. Tin was usually used as a protective layer of the substrate in order to combine a good conductivity, good reliability and a low cost. A pure tin was malleable and reacts with the oxygen to give hard and brittle remains which cause high surface damages. The substrate could be reach and it generates copper oxide remains at the contact surface [11].

Manuscript received July 6, 2018 ; reivsed December 12, 2018.

R. El Abdi and J. Labbéis are with Institut de Physique de Rennes. UMR 6251 UR1 CNRS, Université de Rennes1, 35042 Rennes Cedex, France (e-mail: relabdi@ univ-rennes1.fr).

F. Le Strat is with Entreprise Renault, DEA-TCM, 78084 Guyancourt, France.
In the electrical contact field, fretting-corrosion was an irreversible degradation which avoids a good current conduction. Several analyses were performed to understand this phenomenon and how the current was conducting through the interface for static and dynamic contacts [12, 13]. Due to the many mechanical parameters (force, materials used, type of vibration...), the numerical simulations provide interesting results but cannot predict the real life time of a connector submitted to a fretting-corrosion phenomenon [14].

The aim of this work was to give further information to understand the oxygen influence on the current conduction of a tin-plated contact and oxidized remains. An experimental bench was used to introduce an inert gas at the interface of a tin-plated connector in order to avoid the formation of insulating remains due to the oxygen of the air. The nitrogen gas was chosen during the tests; it was chemically inert for the tin and the copper and it represents $79 \%$ of the atmosphere. Three experimental conditions were realized: samples in air atmosphere, samples submitted to a nitrogen gas flow near the contact interface and samples submitted alternatively to the air and to a nitrogen gas.

\section{EXPERIMENTAL SETUPS}

Two test benches were used. Every bench was composed of a piezo-electric actuator which provides a controlled movement between the clip and the pin. A generator provides a stable current through the interface and the measurements of contact voltage were performed with a voltmeter and a scope for a real-time analysis [12]. The bench was mounted on an anti-vibration table in order to avoid external vibrations.

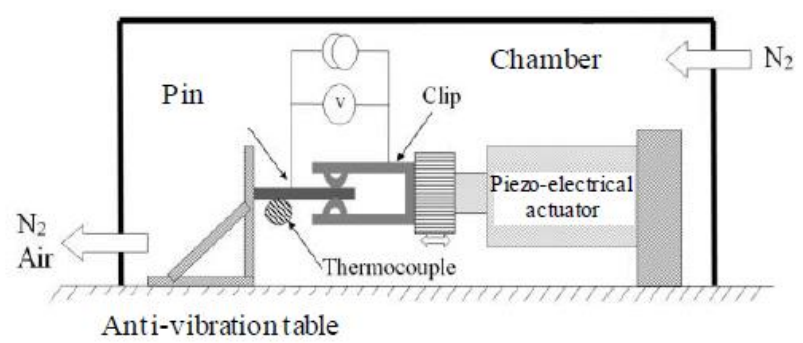

Fig. 1. Schematization of experimental setup: Nitrogen injected into a chamber

The first bench uses a chamber in order to analyze the electrical behavior by introducing the nitrogen gas into a volume of $15.10^{-3} \mathrm{~m}^{3}$ (Fig.1). The second bench uses a Teflon pipe and the nitrogen was injected into a small volume near the contact interface to analyze the electrical behavior (Fig.2). 
The flow was not measured but is sufficiently low to not disturb the test.

The third experiment alternates the air atmosphere and the nitrogen gas flow in order to study the dynamism of contact voltage when the oxidized remains formation was stopped.

Samples were typical terminals of connector. The substrate material was a copper alloy which was plated with a thin layer of tin. The imposed movement has a sinusoidal form. Voltage-Intensity (V-I) measurements were realized from -1 A to +1 A with a step of $100 \mathrm{~mA}$.

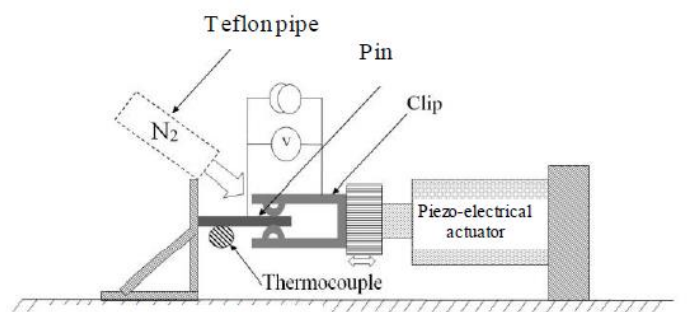

Anti-vibration table

Fig. 2. Schematization of experimental setup: Nitrogen injected near contact surface.

\section{A. Nitrogen Injected Into Chamber}

During this test Fig. 1, the imposed movement has sinusoidal form with amplitude of $10 \mu \mathrm{m}$ peak to peak submitted to a frequency of $20 \mathrm{~Hz}$ when the current value is equal to $200 \mathrm{~mA}$. The nitrogen was introduced 10 minutes before the beginning of the vibration test and thus the oxygen is evacuated from the chamber. Fig. 3 gives the contact voltage curves when vibration tests were made under air atmosphere and under nitrogen atmosphere.

Under the air atmosphere, the contact voltage fluctuates from 500s and increases to reach $0.6 \mathrm{~V}$ around $1500 \mathrm{~s}$. It was a classical electrical behavior of tinned contact [15].

Under a nitrogen atmosphere, the contact voltage remains low along the test Fig. 4 and thus the electrical conduction was perfect but it increases from 0.8 to $1.3 \mathrm{mV}$ between $100 \mathrm{~s}$ and $600 \mathrm{~s}$. It slowly decreases to reach $1 \mathrm{mV}$.

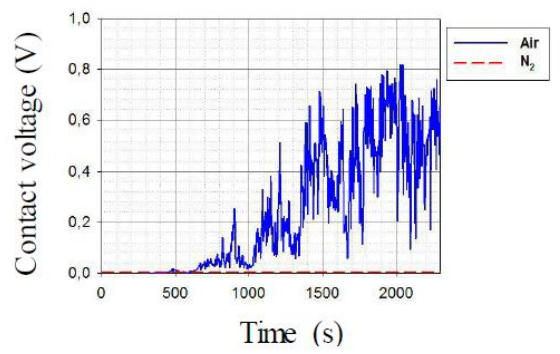

Fig. 3. Contact voltage behavior under air atmosphere and nitrogen.

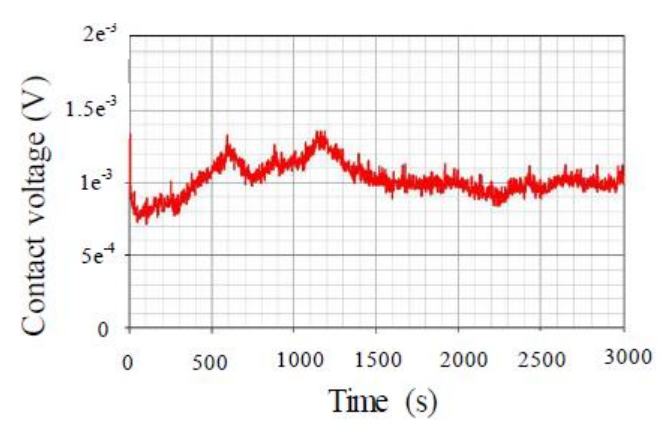

Fig. 4. Zoom of contact voltage under nitrogen atmosphere

Fig. 5 gives the contact voltage at different times when air atmosphere was used. Before $500 \mathrm{~s}$, the curve appearance was linear and thus the electrical conduction was ohmic. After $500 \mathrm{~s}$ the electrical conduction was not ohmic and reaches $0.15 \mathrm{~V}$.

Tests using a nitrogen atmosphere (Fig. 6) showed a linear behavior during the vibration duration. The same curve slopes were obtained with lower values than the one obtained under air atmosphere. Electrical conduction wasn't disturbed when the tests were carried out under a nitrogen atmosphere.

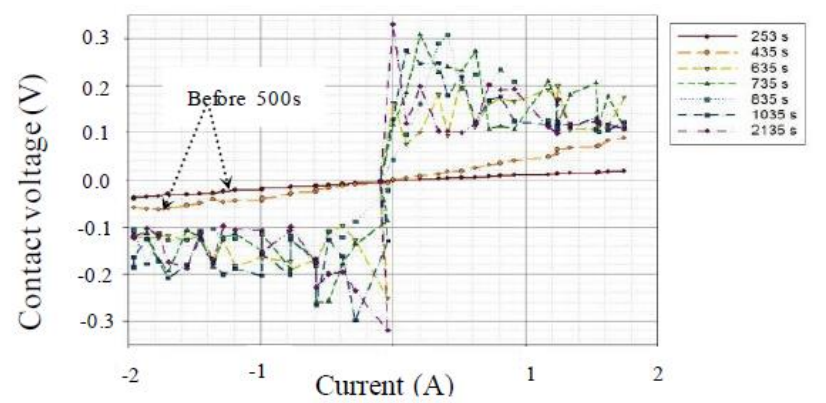

Fig. 5. Contact voltage versus current (test under air atmosphere).

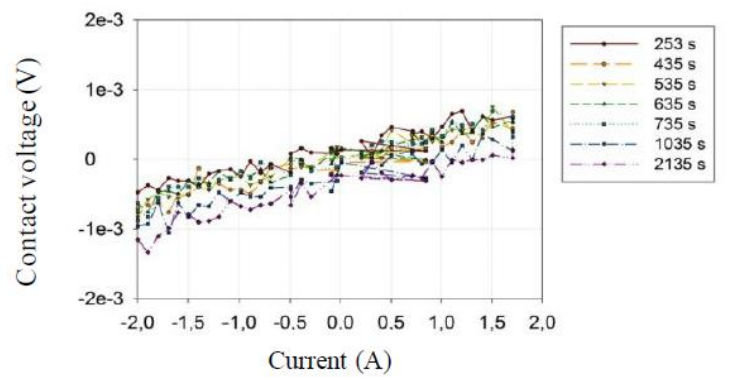

Fig. 6. Contact voltage versus current (test under nitrogen atmosphere).

Under nitrogen atmosphere the contact zone between the pin and the clip (Fig .1) was not vastly damaged (Fig .7a). The contact zone obtained under air atmosphere was severely degraded and many oxidized remains were found around the contact zone ((Fig. 7b).
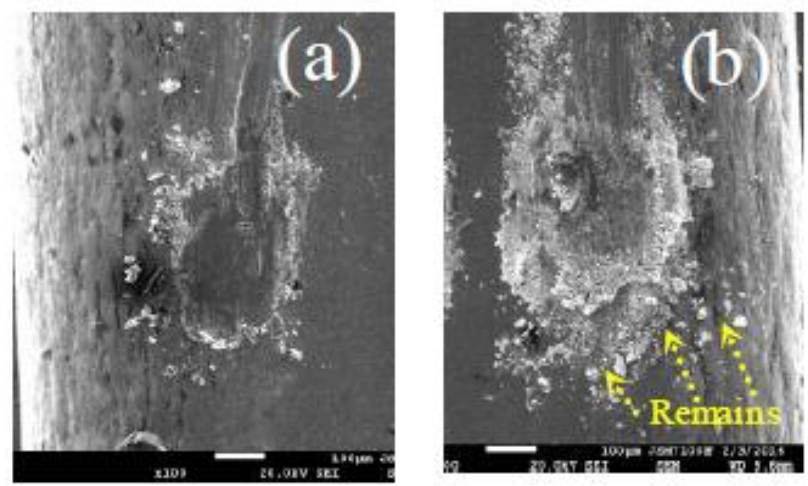

Fig. 7. SEM analysis of contact zone for test under nitrogen atmosphere (a) and for test under air atmosphere (b).

The main idea by using a teflon pipe is to provide a nonoxygen atmosphere as fast as possible but nitrogen gas flow could stop remains oxidation but is not easy to know exactly the moment when the oxidation of remains finishes. A small volume of nitrogen (15 liters) is injected into a chamber. The piezo-electrical actuator produces a movement of $25 \mu \mathrm{m}$ with a frequency of $50 \mathrm{~Hz}$ (current intensity equal to $200 \mathrm{~mA}$ ). Figure 8 gives the contact voltage versus time.

- Up to 250 s, the contact voltage is low. 
- Between 250s and 500s, the contact voltage fluctuates and increases to $0.6 \mathrm{~V}$. At this moment the contact is severely degraded and copper remains were obtained.

- Between 500s and 610s, the contact voltage always fluctuates but decreases to $0.4 \mathrm{~V}$ (hatched zone 1). The injection of nitrogen inside the enclosure leads to a reduction oxygen volume but the formation of oxidized debris is always possible.

- Between 610s and 650s, the contact voltage decreases and become close to zero (hatched area 2). The number of remains decreases and the contact area was less degraded.

- From 650s, the contact voltage becomes small (hatched area 3).

Therefore, it is difficult to define when the exact stop of the oxidation of the debris. Nevertheless, the introduction of nitrogen inside the enclosure leads to a contact voltage close to zero for duration of $150 \mathrm{~s}$. It is likely that oxygen will no longer be present around the contact after $610 \mathrm{~s}$.

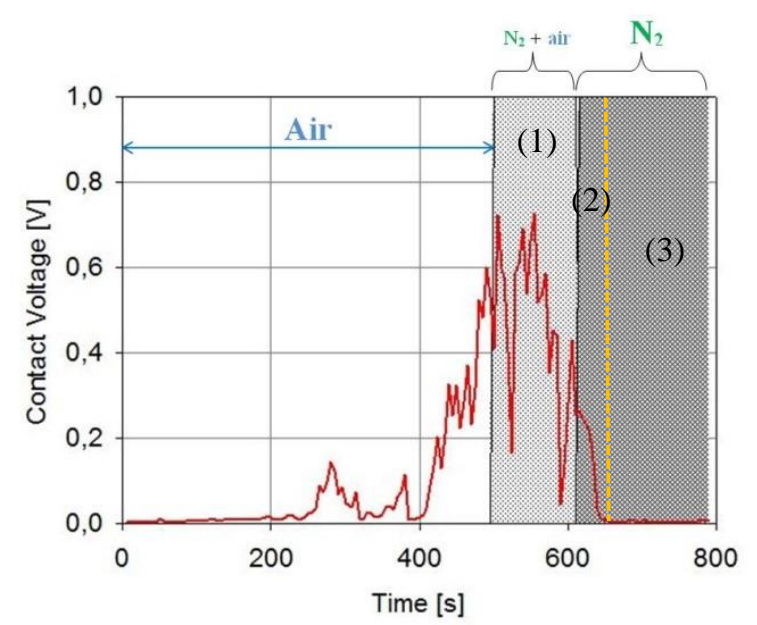

Fig. 8. Contact voltage measurement on a clip/pin system inside a chamber, the nitrogen gas is injected inside the chamber.

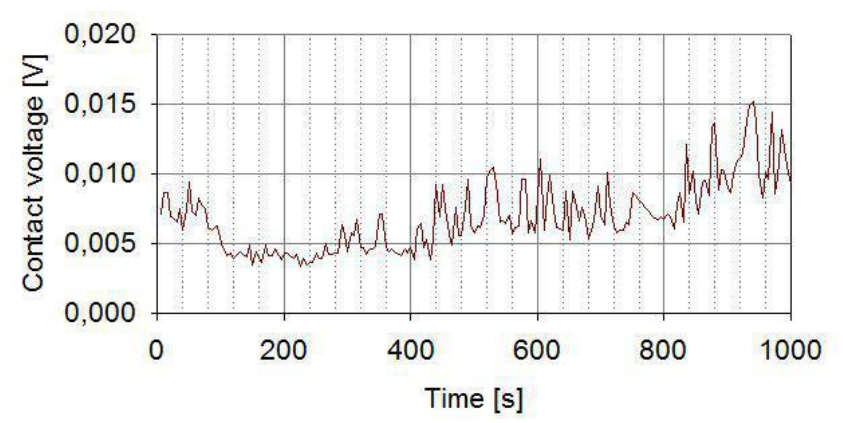

Fig. 9. Contact voltage measurement on a clip/pin system with nitrogen gas flow.

\section{B. Nitrogen Injected Near Contact Surface}

As indicated in Fig. 2, the nitrogen was injected near the contact zone.

Under a continuous neutral gas flow on the sample, the contact tension stays stable with a mean value of $10 \mathrm{mV}$ which indicates a good conductivity during all the experiment. The contact voltage has a $5 \mathrm{mV}$ drop from $80 \mathrm{~s}$ to 120 s then increases slowly until $12 \mathrm{mV}$ (Fig. 9). The injection of nitrogen gas into a small volume i.e. the volume around the interface induced a good conductivity at the interface. Some similarities can be done with a noble metal like the silver as a protective layer [13].

On the other hand, another test was undertaken to alternate simultaneously air and nitrogen gas near the interface of the contact zone. A small volume of air is injected followed by a small volume of nitrogen gas (Fig.10). The imposed movement has a sinusoidal form with amplitude of $60 \mu \mathrm{m}$ peak to peak submitted to a frequency of $50 \mathrm{~Hz}$. Four periods of nitrogen gas injection were performed, four times when the contact voltage decreases instantly until a low and stable value (Fig. 10). The contact voltage increases and quickly fluctuates when the nitrogen gas flow was stopped by the re-introducing oxygen near the interface.

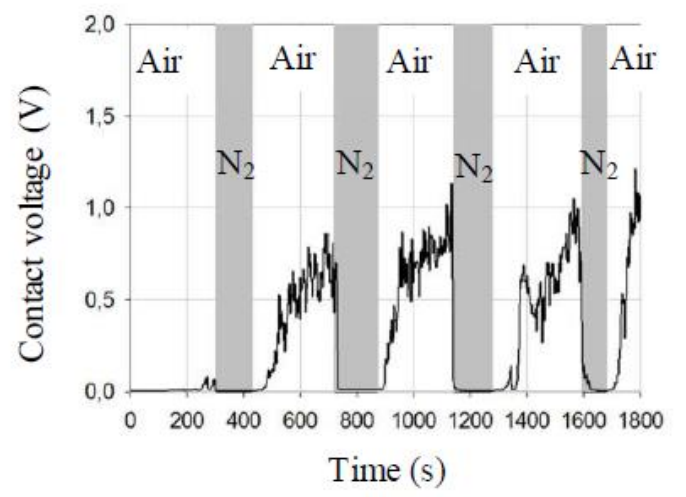

Fig. 10. Contact voltage versus time. Dashed areas indicate nitrogen injection on contact surface during $2 \mathrm{~min}$.

\section{CONCLUSION}

In order to study the fretting-corrosion, tests using the nitrogen gas give interesting results about the role of the remains formation. Under the air atmosphere, the oxidation of remains leads to an increasing of voltage. Under a neutral gas as nitrogen, the contact voltage rapidly drops and this decrease was linked to the evacuation of the remains. After evacuation of the remains and stopping the neutral gas flow, the contact voltage increases instantly due to the reactivity of oxygen, more it's degraded, more their asperities, more the surface of reaction is getting higher and improve the formation of remains.

This type of experiment was undertaken for the first time to study electrical behavior of fretting-corrosion of tin-plated contact. Further experiments should be undertaken by changing some parameters (frequency, amplitude of vibration) in order to get further explanations.

\section{REFERENCES}

[1] C. Chen, G. T. Flowers, M. Bozack, and J. Suhling, "Modeling and analysis of a connector system for prediction of vibration-induced fretting degradation,' IEEE Holm Conference on Electrical Contacts, pp. 129-135, 2009.

[2] J. Labbe, R. E. Abdi, E. Carvou, F. L. Strat, and C. Plouzeau, "Vibration induced at contact point of tighten-up connector system," in Proc. IEEE Holm Conference on Electrical Contacts, pp. 200-204, 2014.

[3] W. H. Abbott, "The development and performance characteristics of mixed flowing gas test environments," in Proc. $33^{\text {rd }}$ IEEE HOLM Conference on Electrical Contacts, pp. 63-78, 1987.

[4] Y. W. Park, H. G. Joo, and K. Y. Lee, "Effect of intermittence fretting on corrosion behavior in electrical contact," Wear, vol. 268, pp. 353360, 2010.

[5] S. Fouvry, P. Kapsa, and L. Vincent, "An elastic-plastic shakedown analysis of fretting wear," Wear, vol. 247, pp.41-54, 2001.

[6] S. El Mossouess, N. Benjemâa, E. Carvou, and R. El Abdi, "Fretting corrosion in power contacts: Electrical and thermal analysis," in Proc. 
$60^{\text {th }}$ IEEE HOLM Conference on Electrical Contacts, pp. 297-301, 2014.

[7] E. Carvou and N. Benjemâa, "Time and level analysis of contact voltage intermittences induced by fretting in power connector," in Proc. $53^{\text {rd }}$ IEEE HOLM Conference on Electrical Contacts, pp. 211$215,2007$.

[8] L. Tristan, "Fiabilisation des connecteurs électriques soumis à des vibrations," PhD thesis, Université Paris-Sud, 2000.

[9] A. Bouzera, E. Carvou, N. Benjemâa, R. El Abdi, L. Tristani, and E. M. Zindine, "Minimum fretting amplitude in medium force for connector coated material and pure metals," in Proc. IEEE Holm Conference on Electrical Contacts, pp. 101-107, 2010.

[10] U. Stocker and G. Bonisch, ATZ Automobiltech, vol. 93, pp. 7-10, 1991.

[11] Y. W. Park, T. S. N. S. Narayanan, and K. Y. Lee, "Fretting corrosion of tin-plated contacts: Evaluation of surface characteristics," Tribology International Journal, vol. 40, pp. 548-559, 2007.

[12] N. Benjemâa and E. Carvou, "Electrical contact behaviour of power connector during fretting vibration," in Proc. IEEE Holm Conference on Electrical Contacts, pp. 263-266, 2006.

[13] P. Jedrzejczyk, S. Fouvry, and P. Chalandon, "Quantitative Description of the Electrical Contact Endurance under Fretting Condition: Comparison between Tin and Silver," IEEE Holm Conference on Electrical Contacts, pp. 272-277, 2008.

[14] S. Tsukiji, S. Sawada, T. Tamai, Y. Hattori, and K. Iida, "Direct observations of current density distribution in contact area light emission diode wafer," in Proc. IEEE Holm Conference on Electrical Contacts, pp. 62-68, 2001.

[15] A. Bouzera, "Fiabilité de la connectique sous sollicitation de vibrations mécaniques," PhD thesis, Université de Rennes , 2012.

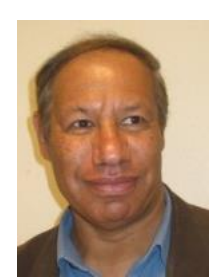

R. El Abdi is a professor at Rennes1 University $\mathbb{I P R}$, UMR 6251 UR1 CNRS- Département Mécanique \&Verres- FRANCE). He is specialized in the structure behavior analysis, in fracture fatigue, damage and mechanical engineering. His current research interests include analysis of numerical methods, finite-element methods, boundary integrals, nonlinear damage modeling and experimental optic fiber analysis. He manages a mechanical team which is interested in surfaces and contact problems. 